\title{
Quantum photonic integrated circuits with waveguide integrated superconducting nanowire single-photon detectors
}

\author{
Gregory Goltsman ${ }^{1,2, *}$ \\ ${ }^{1}$ Department of Physics, Moscow State Pedagogical University, Moscow 119992, Russia \\ ${ }^{2}$ Zavoisky Physical-Technical Institute of the Russian Academy of Sciences, Kazan 420029, Russia
}

\begin{abstract}
We show the design, a history of development as well as the most successful and promising approaches for QPICs realization based on hybrid nanophotonic-superconducting devices, where one of the key elements of such a circuit is a waveguide integrated superconducting single-photon detector (WSSPD). The potential of integration with fluorescent molecules is discussed also.
\end{abstract}

Currently, many physical systems for the realization of qubits including superconducting circuits, trapped ions and atoms, quantum dots, color centers in a solid and photons are intensely studied. Here we focus only on the last item in the list - photons. Because photons interact only weakly with an optically transparent medium but not with each other, have several degrees of freedom for encoding of quantum information and provide fast propagation speed, they are an attractive choice for creating quantum networks. However, while individual photons can be manipulated conveniently, the creation of twoand three- deterministic qubit gates requires strong nonlinear interaction between photons. In this case, the advantages of the photons are compensated for by their shortcomings, which for a long time limited the use of photons as qubits. Fortunately, Knill, Laflamme and Milburn (KLM) proposed the concept of linear optical quantum computing (LOQC), allowing to create non-deterministic gates using photons, linear optical elements and single photon detectors. While the implementation of the KLM-protocol is possible in free space, the need for a large number of optical components and their precise configuration requires a more complex solutions. Due to a number of advantages, such as scalability, small footprint, inherent stability, no need for optical alignment as well as low power consumption and CMOS-compatibility, quantum photonic integrated circuits (QPICs) can successfully overcome these challenges.

Popular material platforms for the realization of QPICs include silicon, gallium arsenide, and diamond. Each platform has its own advantages and disadvantages and is currently in different stages of development [1]. Nevertheless, all of the platforms rely on combining single photon sources, linear optical elements and single-photon detectors on chip.

\footnotetext{
* Corresponding author: goltsman@rplab.ru
} 
Recently, a fully integrated circuit including a single photon source (carbon nanotube), detectors (superconducting nanowire single-photon detectors) and silicon-nitride waveguides has already been implemented on a single chip [2]. Despite the demonstration of the working concept, the purity of the emulsion of electroluminescent photons is not yet sufficient for large scale processing and new single-photon sources are constantly being investigated.

a)

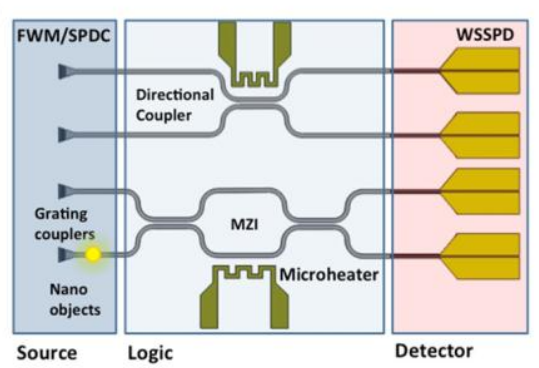

b)

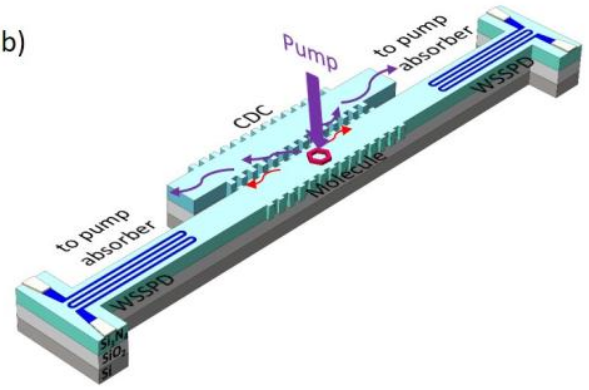

Fig. 1. a) Schematic view of the platform for the realization of QPIC. The nanophotonic chip includes single-photon sources, single photon detectors and quantum logic. FWM is four wave mixing and SPDC is spontaneous parametric down-conversion. b) Schematic view of a nanophotonic circuit with on-chip fluorescent single molecule

Fluorescent molecules are one of the promising single-photon sources. Recently the emission of single photons from Dibenzoterrylene (DBT) molecules into $\mathrm{Si}_{3} \mathrm{~N}_{4}$ ridge waveguide at room temperature was demonstrated. DBT was placed on a $\mathrm{Si}_{3} \mathrm{~N}_{4}$ waveguide to couple emitted single photons through the evanescent waveguide mode into the guided mode. The advantages of this approach include a small footprint, simple fabrication methods and scalability towards arrays of integrated single photon sources for QPICs. However, unlike the on-chip integrated electroluminescent nanotube [3], a more complex photonic circuit is required. The next step in the integration of a single-photon emitted molecule and QPIC including effective pump rejection (CDC) and superconducting nanowire single-photon detectors (WSSPDs) is proposed in Fig. 1b. As an efficient on-chip filter a contra-directional coupler (CDC) can be used. After optical pumping of a single molecule, the residual pump is rejected to the neighboring waveguide and routes to the optical absorber. Emitted photons are directed to WSSPD, reproducing the Hanbury Brown-Twiss scheme. By measuring the on-chip second order correlation function, one can detect non-classical light for QPICs, as well as to study the molecules itself.

Going beyond such proof-of-principle concepts, the realization of large scale QPICs is expected to have profound impact on science and technology, material engineering, as well as quantum information processing including quantum computing, simulation and metrology.

G. Goltsman acknowledges support by Ministry of Education and Science of the Russian Federation № 14.583.21.0065.

\section{References}

1. S. Bogdanov, M. Y. Shalaginov, A. Boltasseva, A. Shalaev. Opt. Mater. Express 7, 111 (2016)

2. S. Khasminskaya, F. Pyatkov, K. Słowik, S. Ferrari, O. Kahl, V. Kovalyuk, P. Rath, A. Vetter, F. Hennrich, M. M. Kappes, G. Gol'tsman, A. Korneev, C. Rockstuhl, R. Krupke, and W. H. P. Pernice. Nat. Photonics 10, 727 (2016) 